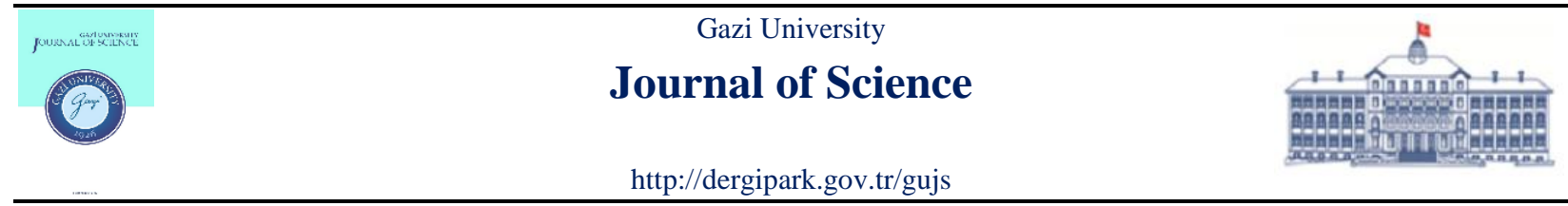

\title{
Approximate Bound State Solutions of the Hellmann Plus Kratzer Potential in N-dimensional Space
}

\author{
Aysel OZFIDAN * \\ Tarsus University, Department of Natural and Mathematical Sciences, 33400, Mersin, Turkey
}

Highlights

- We probe the N-dimensional approximate solutions of the Hellmann plus Kratzer potential.

- The graphical perspective for consideration potential has been presented with Matlab software.

- We analyzed the accuracy of the asymptotic iteration formalism.

\section{Article Info}

Received: 09/01/2020

Accepted: 19/03/2020

\section{Keywords}

\section{Hellmann}

Kratzer

$N$-dimensions

\begin{abstract}
We have examined the approximate $l_{N-1}$-state solutions of the $\mathrm{N}$-dimensional Schrödinger equation for a particle interacting with the Hellmann plus Kratzer potential. In hyperspherical coordinate system, we have constructed the bound state energy equation and the wavefunctions expressed by the hypergeometric function via the asymptotic iteration approach in detail. When considered the special cases of parameters in Hellmann plus Kratzer potential, this potential turns into several potential models. In this connection, the non-relativistic energy spectra for the modified Kratzer, Yukawa, Coulomb and Hellmann potentials in approximate analytic form have been obtained in hyperspherical coordinates. We have presented the numerical energy eigenvalues for the Hellmann, Yukawa and Coulomb potentials in $N=3$ dimensions. Our present results provide an appropriate test of the accuracy of asymptotic iteration formalism.
\end{abstract}

\section{INTRODUCTION}

The interaction of quantum systems with the spherically symmetric fields have been attracted the attention of researchers since the dawn of quantum mechanics. The solutions of the N-dimensional Schrödinger equation for central potentials including centrifugal term are also of great interest. In establishing solutions for the exponential types-potential models, we have to use some approximations such as Greene-Aldrich, Pekeris. Our work address the problem of the construction of the N-dimensional rovibrational energy spectrum and radial wave functions for Hellmann plus Kratzer potential in the presence of Greene-Aldrich approximation scheme [1]. The solution methodology considered in this paper is based on asymptotic iteration method(AIM) proposed by Ciftci et al. [2-4].

Hyperspherical coordinate system is considerably useful for analyzing the quantum mechanical problem. In this connection, non-relativistic aspect of the N-dimensional bound state solutions for Hellmann plus Kratzer potential model has been reviewed in this present work. It should be mentioned that mathematical tools have been examined by Louck and Shaffer [5], Louck [6,7] and Chatterjee [8] to generalize the orbital angular momentum. In hyperspherical coordinates, Hellmann plus Kratzer potential can be expressed as

$V_{N}(r)=-\frac{V_{0}}{r}+\frac{V_{1}}{r} e^{-\delta r}+D_{e}\left(\frac{r-r_{e}}{r}\right)^{2}$ 
where $V_{0}$ and $V_{1}$ are the strength of the potential, $\delta$ is the potential parameter, $r_{e}$ and $D_{e}$ are the equilibrium intermolecular seperation, dissociation energy, respectively. In Equation (1), if we consider the potential parameters as follows

- $D_{e}=0$, the potential turns to Hellmann potential,

- $V_{0}=0$ and $V_{1}=0$, it will reduce to the modified Kratzer potential,

- $D_{e}=0$ and $V_{0}=0$, Equation (1) transforms to Yukawa potential,

- $D_{e}=0$ and $V_{1}=0$, this potential becomes Coulomb potential.

Hellmann potential which is a combination of the attractive Coulomb and Yukawa potentials is used to analyze the systemization of energyeigenvalues. This potential with $V_{1}>0$ was firstly introduced by Hellmann[9-10] and then has been used to study for the case of positive and negative $V_{1}$. Kratzer potential was proposed by Kratzer[11] in 1920 because of its importance in the description of molecular structures and interactions of diatomic molecules model. Subsequently, this potential has played a vital role in the realm of molecular physics and quantum chemistry. In the framework of non-relativistic and relativistic theory, extensive research has been carried out on Hellmann potential and Kratzer potential in literature[1215]. We present the graphical perspective of the Hellmann plus Kratzer potential with Matlab software. By considering $V_{0}=4, V_{1}= \pm 15, D_{e}=6, r_{e}=1.65$, we have plotted the $3 \mathrm{D}$ graphics for Hellmann and Hellmann plus Kratzer potential. We remark that the effects of the existence and absence of the modified Kratzer potential have been investigated in Figures 1 and 2. In Figures1 and 2, the distance $r$ and the parameter $\delta$ are in the range of 0.1-6, 0.1-0.5, respectively. In the case of $V_{1}<0$, the effect of the modified Kratzer potential clearly have been observed in Figure 1(b). However, in Figure 2, the surface plots of Hellmann potential and Hellmann plus Kratzer potential have similar behaviour because the value of $V_{1}$ has positive.

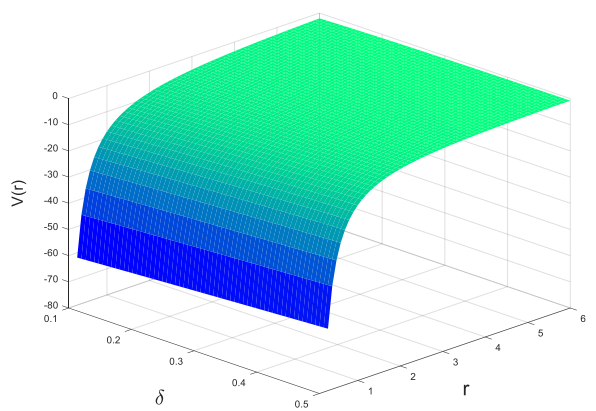

(a)

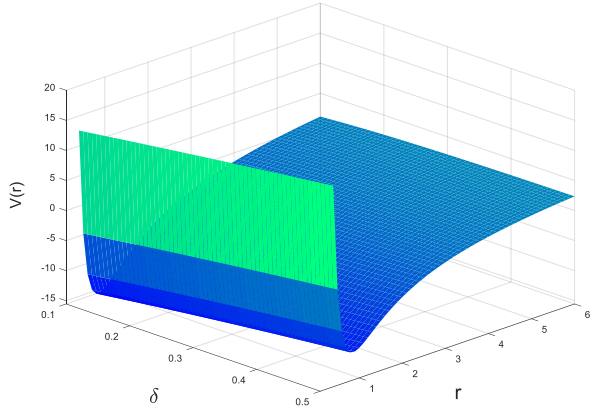

(b)

Figure 1. In the case of $V_{1}<0$, the surface plots of (a) Hellmann potential and (b) Hellmann plus Kratzer potential as a function of $\delta$ and $r$

In spherical coordinates, various forms of the potential given in Equation (1) have been examined by using different approaches such as the perturbation, variatonal, parametric NU and AIM[14-23]. On the other hand, by applying the orthogonal polynomial solution method[24], AIM[25], NU[26], researchers have studied the N-dimensional solutions of Schrödinger equation under Coulomb, the modified Kratzer potential which are the the special cases of Hellmann plus Kratzer potential. To our knowledge, the asymptotic iteration formalism has not been applied to solve the N-dimensional Schrödinger equation with the Hellmann plus Kratzer potential. In this context, we focus on the approximate analytic solutions of this 
potential in hyperspherical coordinates through the asymptotic iteration approach. This approach of solution is based on solving the second-order homogeneous linear differential equations. AIM is an alternative solution method because it is systematic and efficient.

The main pillars of our work is as follows. Section 2 is devoted to the general view of asymptotic iteration method. In Section 3, we present the N-dimensional solutions of the Schrödinger particle interacting with the Hellmann plus Kratzer potential within the framework of Greene-Aldrich approximation and AIM. In Section 4, by considering different cases of the potential parameters, we introduce the energy equations for the special forms of this potential in hyperspherical coordinates. Besides, numerical results for energy spectra generated by Hellmann, Yukawa and Coulomb potentials are presented. Afterall, Section 5 contains the concluding remarks.

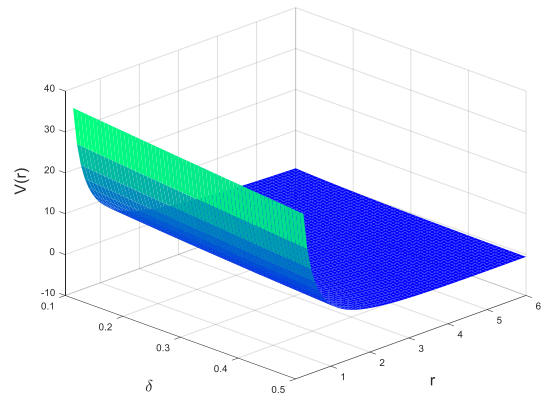

(a)

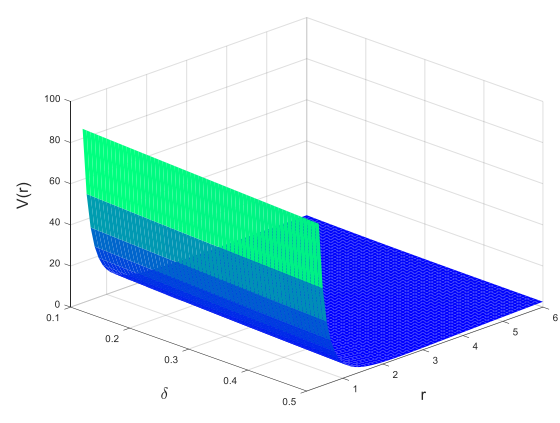

(b)

Figure 2. In the case of $V_{1}>0$, the surface plots of (a) Hellmann potential and (b) Hellmann plus Kratzer potential as a function of $\delta$ and $r$

\section{APPROXIMATE BOUND STATE SOLUTIONS FOR THE HELLMANN PLUS KRATZER POTENTIAL}

The motion of a particle in the spherically symmetric potential field in $\mathrm{N}$-dimensions is described within the framework of non-relativistic formalism as follows

$$
\left[-\frac{\hbar^{2}}{2 \mu} \nabla_{N}^{2}+V_{N}(r)\right] \psi(\vec{r})=E \psi(\vec{r})
$$

where $E$ and $\mu$ are non-relativistic energy and reduced mass, $V_{N}(r)$ is the N-dimensional central potential, $\hbar$ is Planck constant.

The Laplacian in N-dimensions is defined with respect to Cartesian coordinates $x_{1}, x_{2}, x_{3} \ldots x_{N}$ as

$$
\nabla_{N}^{2}=\sum_{j=1}^{N} \frac{\partial^{2}}{\partial x_{j}^{2}}
$$

To begin with, we need to present the hyperspherical coordinates in $\mathrm{N}$-dimensional space based on Louck[6] and Chatterjee's works[8] as follows 


$$
\begin{aligned}
& x_{1}=r \cos \theta_{1} \sin \theta_{2} \sin \theta_{3} \ldots \sin \theta_{N-1} \\
& x_{2}=r \sin \theta_{1} \sin \theta_{2} \sin \theta_{3} \ldots \sin \theta_{N-1} \\
& x_{3}=r \cos \theta_{2} \sin \theta_{3} \sin \theta_{4} \ldots \sin \theta_{N-1} \\
& x_{4}=r \cos \theta_{3} \sin \theta_{4} \sin \theta_{5} \ldots \sin \theta_{N-1} \\
& \quad \vdots \\
& x_{j}=r \cos \theta_{j-1} \sin \theta_{j} \sin \theta_{j+1} \ldots \sin \theta_{N-1}, \quad 3 \leq j \leq N-1 \\
& x_{N}=r \cos \theta_{N-1}
\end{aligned}
$$

for $N=3,4,5 \ldots$ where the range of variable is $0 \leq r \leq \infty, 0 \leq \theta_{1} \leq 2 \pi, 0 \leq \theta_{j} \leq \pi$ for $j=2,3, \ldots N-$ 1 and $r$ is the radius of an $\mathrm{N}$-dimensional sphere. In connection with the hyperspherical coordinates, the Laplacian has the form

$$
\begin{aligned}
& \nabla_{N}^{2}=\frac{1}{r^{N-1}} \frac{\partial}{\partial r} r^{N-1} \frac{\partial}{\partial r}+\frac{1}{r^{2}} \sum_{k=1}^{N-2} \frac{1}{\sin ^{2} \theta_{k+1} \sin ^{2} \theta_{k+2} \ldots \sin ^{2} \theta_{N-1}}\left(\frac{1}{\sin ^{k-1} \theta_{k}} \frac{\partial}{\partial k} \sin ^{k-1} \theta_{k} \frac{\partial}{\partial \theta_{k}}\right) \\
& +\frac{1}{r^{2}}\left(\frac{1}{\sin ^{N-2} \theta_{N-1}} \frac{\partial}{\partial \theta_{N-1}} \sin ^{N-2} \theta_{N-1} \frac{\partial}{\partial \theta_{N-1}}\right) .
\end{aligned}
$$

By taking the hyperspherical total wavefunction as

$\psi\left(r, \theta_{1}, \theta_{2}, \ldots, \theta_{N-1}\right)=r^{-\frac{N-1}{2}} R(r) Y_{l_{N-1}, l_{N-2}, \ldots l_{2}, l_{1}}\left(\theta_{1}, \theta_{2}, \ldots \theta_{N-1}\right)$

in which $R(r)$ and $Y_{l_{N-1}, l_{N-2}, \ldots l_{2}, l_{1}}\left(\theta_{1}, \theta_{2}, \ldots \theta_{N-1}\right)$ are the radial wavefunction, the generalized spherical harmonics, respectively and employing the way of separating variables, we obtain the non-relativistic wave equation in hyperspherical coordinates for the function $R(r)$

$\frac{d^{2} R(r)}{d r^{2}}+\left[\frac{2 \mu}{\hbar^{2}}(E-V(r))-\frac{(N-1)(N-3)}{4 r^{2}}-\frac{l_{N-1}\left(l_{N-1}+N-2\right)}{r^{2}}\right] R(r)=0$.

The eigenvalue equation of the generalized angular momentum operator is also given by

$$
\begin{aligned}
& L_{N-1}^{2} Y_{l_{N-1}, l_{N-2}, \ldots l_{2}, l_{1}}\left(\theta_{1}, \theta_{2}, \ldots \theta_{N-1}\right)= \\
& l_{N-1}\left(l_{N-1}+N-2\right) \hbar^{2} Y_{l_{N-1}, l_{N-2}, \ldots l_{2}, l_{1}}\left(\theta_{1}, \theta_{2}, \ldots \theta_{N-1}\right) .
\end{aligned}
$$

It should be mentioned that the more detailed information about the generalized angular momentum operators can be found in $[6,8]$.

\subsection{N-dimensional Energy Spectrum In Approximate Analytic Form}

We now examine solutions of the radial Schrödinger equation with the Hellmann plus Kratzer potential in $\mathrm{N}$-dimensions by means of the asymptotic iteration method. First of all, when we substitute Equation (1) into Equation (7), we obtain the radial Schrödinger equation for the Hellmann plus Kratzer potential in hyperspherical coordinates as

$$
\frac{d^{2} R(r)}{d r^{2}}+\left[\frac{2 \mu}{\hbar^{2}}\left(E+\frac{V_{0}}{r}-\frac{V_{1}}{r} e^{-\delta r}-D_{e}\left(\frac{r-r_{e}}{r}\right)^{2}\right)-\frac{(N-1)(N-3)}{4 r^{2}}-\frac{l_{N-1}\left(l_{N-1}+N-2\right)}{r^{2}}\right] R(r)=0 .
$$


Equation (9) cannot be solved analytically for any $\boldsymbol{l}_{\boldsymbol{N}-\mathbf{1}}$-state on account of the presence of the centrifugal term. For this reason, an approximation has to be used in order to solve this equation. Proposed by Greene and Aldrich [1], an approximation scheme which is a good approximation to centrifugal term in short range potential as pointed out in [27] is written as follows

$\frac{1}{r^{2}} \approx \frac{\delta^{2}}{\left(1-e^{-\delta r}\right)^{2}}$

which is valid for $\delta \ll 1$. If we apply this approximation and transformation $y=e^{-\delta r}$ to Equation (9), we rewrite the hyperradial wave equation in non-relativistic theory

$\frac{d^{2} R(y)}{d y^{2}}+\frac{1}{y} \frac{d R(y)}{d y}+\left[-\frac{\varepsilon^{2}}{y^{2}}+\frac{\gamma+v}{y^{2}(1-y)}+\frac{\Lambda}{y(1-y)}-\frac{\eta}{y^{2}(1-y)^{2}}\right] R(y)=0$

where

$-\varepsilon^{2}=\frac{2 \mu}{\hbar^{2}}\left(E-D_{e}\right), \kappa=l_{N-1}+\frac{N-3}{2}, \gamma=\frac{4 \mu D_{e} r_{e}}{\hbar^{2} \delta}$

$\Lambda=-\frac{2 \mu V_{1}}{\hbar^{2} \delta}, \nu=\frac{2 \mu V_{0}}{\hbar^{2} \delta}, \eta=\frac{2 \mu D_{e} r_{e}^{2}}{\hbar^{2}}+\kappa(\kappa+1)$

To solve Equation (11) via asymptotic iteration approach, the following acceptable physical wavefunction is proposed

$R(y)=(1-y)^{\rho+1} y^{\beta} f(y)$

where $f(y)$ is a function to be determined, $\rho$ and $\beta$ are defined as

$\rho=-\frac{1}{2}+\frac{1}{2}(1+4 \eta)^{\frac{1}{2}}, \beta=\left(\varepsilon^{2}-(\gamma+v)+\eta\right)^{\frac{1}{2}}$

If this proposed wavefunction is substituted into Equation (11), the following second-order homogenous linear differential equation is obtained

$\frac{d^{2} f(y)}{d y^{2}}=\left[\frac{(2 \beta+2 \rho+3) y-(2 \beta+1)}{y(1-y)}\right] \frac{d f(y)}{d y}+\left[\frac{(1+\rho)(2 \beta+1)+(2 \eta-(\gamma+v))-\Lambda}{y(1-y)}\right] f(y)$

which is easily handled to AIM solutions. As well known, Equation (15) is called the hypergeometric differential equation. By employing functional analysis method, we can probe the solutions of this differential equation. However, the solution methodology considered in our study is based on the asymptotic iteration approach.

If Equation (2) is taken into consideration, the expressions of $\lambda_{0}$ and $s_{0}$ are determined. With Equation (A.4), it is then easy to obtain the values of $\lambda_{n}(y)$ and $s_{n}(y)$ in the following forms

$\lambda_{0}=\frac{(2 \beta+2 \rho+3) y-(2 \beta+1)}{y(1-y)}$
$s_{0}=\frac{(1+\rho)(2 \beta+1)+(2 \eta-(\gamma+v))-\Lambda}{y(1-y)}$ 


$$
\begin{aligned}
& \lambda_{1}=\frac{2 \rho+2 \beta+3}{y(1-y)}-\frac{(2 \rho+2 \beta+3) y-(2 \beta+1)}{y^{2}(1-y)}+\frac{(2 \rho+2 \beta+3) y-(2 \beta+1)}{y(1-y)^{2}} \\
& +\frac{(1+\rho)(2 \beta+1)+2 \eta-(\beta+v)-\Lambda}{y(1-y)}+\frac{((2 \rho+2 \beta+3) y-(2 \beta+1))^{2}}{y^{2}(1-y)^{2}} \\
& S_{1}=\frac{(1+\rho)(2 \beta+1)+(2 \eta-(\gamma+v))-\Lambda}{y^{2}(1-y)}+\frac{(1+\rho)(2 \beta+1)+(2 \eta-(\gamma+v))-\Lambda}{y(1-y)^{2}} \\
& +\frac{((1+\rho)(2 \beta+1)+(2 \eta-(\gamma+v))-\Lambda)((2 \beta+2 \rho+3) y-(2 \beta+1))}{y^{2}(1-y)^{2}}
\end{aligned}
$$

In order to calculate the radial energy eigenvalues in hyperspherical coordinates, the termination condition given by Equation (A.3) is used. Hence, these energy eigenvalues are obtained as follows

$$
\begin{aligned}
& \frac{s_{0}}{\lambda_{0}}=\frac{s_{1}}{\lambda_{1}} \Rightarrow \Lambda_{0}=2 \beta \rho+2 \beta+1+\rho+2 \eta-(\gamma+v) \\
& \frac{s_{1}}{\lambda_{1}}=\frac{s_{2}}{\lambda_{2}} \Rightarrow \Lambda_{1}=2 \beta \rho+4 \beta+4+3 \rho+2 \eta-(\gamma+v) \\
& \frac{s_{2}}{\lambda_{2}}=\frac{s_{3}}{\lambda_{3}} \Rightarrow \Lambda_{2}=2 \beta \rho+6 \beta+9+5 \rho+2 \eta-(\gamma+v) \\
& \vdots
\end{aligned}
$$

When the above expressions are generalized, the eigenvalues turn out as

$$
\Lambda_{n}=2 \beta(\rho+n+1)+(2 n+1) \rho+(n+1)^{2}+2 \eta-(\gamma+v), n=0,1,2, \ldots
$$

If we substitute the used abbrevations into Equation (18), we obtain the rovibrational energy spectrum for the Hellmann plus Kratzer potential in hyperspherical coordinates

$$
\begin{aligned}
& E_{n l_{N-1}}=D_{e}+\frac{\hbar^{2} \delta^{2}}{2 \mu}\left[\frac{\left(2 l_{N-1}+N-3\right)\left(2 l_{N-1}+N-1\right)}{4}+\frac{2 \mu D_{e} r_{e}^{2}}{\hbar^{2}}-\frac{2 \mu\left(V_{0}+2 D_{e} r_{e}\right)}{\hbar^{2} \delta}\right] \\
& -\frac{\hbar^{2} \delta^{2}}{2 \mu}\left[\frac{\frac{4 \mu D_{e} r_{e}+2 \mu\left(V_{0}-V_{1}\right)}{\hbar^{2} \delta}-\frac{4 \mu D_{e} r_{e}^{2}}{\hbar^{2}}-\frac{\left(2 l_{N-1}+N-3\right)\left(2 l_{N-1}+N-1\right)}{2}-(n+1)^{2}-\left(n+\frac{1}{2}\right)\left(-1+\sqrt{1+\frac{8 \mu D_{e} r_{e}^{2}}{\hbar^{2}}+\left(2 l_{N-1}+N-3\right)\left(2 l_{N-1}+N-1\right)}\right)}{2 n+1+\sqrt{1+\frac{8 \mu D_{e} r_{e}^{2}}{\hbar^{2}}+\left(2 l_{N-1}+N-3\right)\left(2 l_{N-1}+N-1\right)}}\right]
\end{aligned}
$$

In spherical coordinates, this energy spectrum coincides with the one obtained previously in [23].

\section{2. $\mathrm{N}$-dimensional Wavefunctions}

In this section, we establish the $\mathrm{N}$-dimensional wavefunctions related with the rovibrational energy eigenvalues found in the foregoing part. The corresponding eigenfunctions of the hyperradial Schrödinger equation with Hellmann plus Kratzer potential can be derived from the wavefunction generator given by Equation (A.6) 


$$
\begin{aligned}
& f_{0}(y)=C_{2}=C_{2}{ }_{2} F_{1}(0,2 \rho+2 \beta+2,2 \beta+1, y) \\
& f_{1}(y)=-C_{2}[2 \beta+1-(2 \rho+2 \beta+3) y] \\
& =-C_{2}(2 \beta+1){ }_{2} F_{1}(-1,2 \rho+2 \beta+3,2 \beta+1, y)
\end{aligned}
$$

$$
\begin{aligned}
& f_{2}(y)=C_{2}\left[(2 \beta+1)(2 \beta+2)-2(2 \rho+2 \beta+4)(2 \beta+2) y+(2 \rho+2 \beta+4)(2 \rho+2 \beta+5) y^{2}\right] \\
& =C_{2}(2 \beta+1)(2 \beta+2){ }_{2} F_{1}(-2,2 \rho+2 \beta+4,2 \beta+1, y) \\
& f_{3}(y)=-C_{2}[(2 \beta+1)(2 \beta+2)(2 \beta+3)-3(2 \rho+2 \beta+5)(2 \beta+3)(2 \beta+2) y \\
& \left.3(2 \rho+2 \beta+6)(2 \rho+2 \beta+5)(2 \beta+3) y^{2}-(2 \rho+2 \beta+7)(2 \rho+2 \beta+6)(2 \rho+2 \beta+5) y^{3}\right] \\
& =-C_{2}(2 \beta+1)(2 \beta+2)(2 \beta+3){ }_{2} F_{1}(-3,2 \rho+2 \beta+5,2 \beta+1, y)
\end{aligned}
$$

$\vdots$

According to these results, the wavefunction $f(y)$ can be generalized given below

$$
f_{n}(y)=C_{2}(2 \beta+1)_{n}(-1)^{n}{ }_{2} F_{1}(-n, 2 \rho+2 \beta+2+n, 2 \beta+1, y)
$$

in which ${ }_{2} F_{1}$ represents the Gauss hypergeometric function being defined as

$$
{ }_{2} F_{1}(-n, b, c, x)=\sum_{k=0}^{n} \frac{(-n)_{k}(b)_{k} x^{k}}{(c)_{k} k !}
$$

the Pochhammer symbol $(\alpha)_{k}$ is defined by $(\alpha)_{0}=1$ and $(\alpha)_{k}=\alpha(\alpha+1)(\alpha+2) \ldots(\alpha+k-1)=$ $\frac{\Gamma(\alpha+k)}{\Gamma(\alpha)}$ for $k=1,2,3 \ldots$ Hence, the unnormalized hyperradial wavefunction for the Hellmann plus Kratzer potential is

$R(y)=(1-y)^{\rho+1} y^{\beta} C_{2}(2 \beta+1)_{n}(-1)^{n}{ }_{2} F_{1}(-n, 2 \rho+2 \beta+2+n, 2 \beta+1, y)$

in which $C_{2}$ denotes the integration constant that can be built by normalization condition. Then, when we substitute $y=e^{-\delta r}$ into Equation (22) and we use the relation between the hypergeometric function and the Jacobi polynomials [28], we obtain in the following form

$R(r)=C_{2}\left(1-e^{-\delta r}\right)^{\rho+1} e^{-\delta \beta r} n ! P_{n}^{(2 \beta, 2 \rho+1)}\left(1-2 e^{-\delta r}\right)$.

In order to find out this constant, we also benefit from the following two integrals [28]

$$
\begin{aligned}
& \int_{-1}^{1}(1-x)^{\alpha-1}(1+x)^{\beta}\left[P_{n}^{(\alpha, \beta)}(x)\right]^{2} d x=2^{\alpha+\beta} \frac{\Gamma(n+\alpha+1) \Gamma(n+\beta+1)}{n ! \alpha \Gamma(n+\alpha+\beta+1)} \\
& \int_{-1}^{1}(1-x)^{\alpha}(1+x)^{\beta}\left[P_{n}^{(\alpha, \beta)}(x)\right]^{2} d x=2^{\alpha+\beta+1} \frac{\Gamma(n+\alpha+1) \Gamma(n+\beta+1)}{n ! \Gamma(n+\alpha+\beta+1)(2 n+\alpha+\beta+1)}
\end{aligned}
$$

and the normalized radial wavefunction for Hellmann plus Kratzer potential is determined as

$$
\begin{aligned}
& R(r)=\left(1-e^{-\delta r}\right)^{\rho+1} e^{-\delta \beta r}\left[\frac{2 \beta(n+\rho+\beta+1) n ! \Gamma(2 \rho+2 \beta+2+n) \delta}{(n+\rho+1) \Gamma(2 \rho+2+n) \Gamma(2 \beta+1+n)}\right]^{1 / 2} \\
& \times P_{n}^{(2 \beta, 2 \rho+1)}\left(1-2 e^{-\delta r}\right) .
\end{aligned}
$$


For $N=3$, this hyperradial wavefunction consistents with the one investigated by Edet et al.[23] using Nikiforov-Uvarov method.

\section{SPECIAL CASES OF HELLMANN PLUS KRATZER POTENTIAL}

\subsection{Hellmann Potential}

In the case of $D_{e}=0$, the potential given in Equation (1) has the form

$V_{N}(r)=-\frac{V_{0}}{r}+\frac{V_{1}}{r} e^{-\delta r}$

In this limit, we can find the $\mathrm{N}$-dimensional rovibrational energy spectrum for Hellmann potential determined from Equation (19) as follows

$$
\begin{aligned}
& E_{n l_{N-1}}=\frac{\hbar^{2} \delta^{2}}{2 \mu}\left[\frac{\left(2 l_{N-1}+N-3\right)\left(2 l_{N-1}+N-1\right)}{4}-\frac{2 \mu V_{0}}{\hbar^{2} \delta}\right] \\
& -\frac{\hbar^{2} \delta^{2}}{2 \mu}\left[\frac{\frac{2 \mu\left(V_{0}-V_{1}\right)}{\hbar^{2} \delta}-\frac{\left(2 l_{N-1}+N-3\right)\left(2 l_{N-1}+N-1\right)}{2}-(n+1)^{2}-\left(n+\frac{1}{2}\right)\left(-1+\sqrt{1+\left(2 l_{N-1}+N-3\right)\left(2 l_{N-1}+N-1\right)}\right)}{2 n+2 l_{N-1}+N-1}\right]^{2} .
\end{aligned}
$$

For $N=3$, we point out that Equation (26) transforms to the non-relativistic energy spectrum for Hellmann potential in three dimensions obtained by Hamzavi et al.[14].

\subsection{The Modified Kratzer Potential}

When the screening parameter $(\delta)$ goes to zero and also $V_{0}=0, V_{1}=0$, Hellmann plus Kratzer potential turns to the modified Kratzer potential in the following form

$V_{N}(r)=D_{e}\left(\frac{r-r_{e}}{r}\right)^{2}$

and the energy equation which is given in Equation (19) becomes the energy spectrum for the modified Kratzer potential in hyperspherical coordinates as follows

$$
E_{n l_{N-1}}=D_{e}-\frac{8 \mu D_{e}^{2} r_{e}^{2}}{\left(2 n+1+\sqrt{1+\frac{8 \mu D_{e} r_{e}^{2}}{\hbar^{2}}+\left(2 l_{N-1}+N-3\right)\left(2 l_{N-1}+N-1\right)}\right)^{2}}
$$

We report that this non-relativistic energy spectrum agrees with the result of [25].

\subsection{Yukawa Potential}

If the parameters $D_{e}$ and $V_{0}$ equal to zero, Equation (1) transforms to Yukawa potential in N-dimensions which is defined as

$V_{N}(r)=\frac{V_{1}}{r} e^{-\delta r}$ 
and so we can obtain the $\mathrm{N}$-dimensional energy spectrum for quantum systems with Yukawa potential in the non-relativistic theory

$$
\begin{aligned}
& E_{n l_{N-1}}=\frac{\hbar^{2} \delta^{2}}{2 \mu}\left[\frac{\left(2 l_{N-1}+N-3\right)\left(2 l_{N-1}+N-1\right)}{4}\right] \\
& -\frac{\hbar^{2} \delta^{2}}{2 \mu}\left[\frac{\frac{-2 \mu V_{1}}{\hbar^{2} \delta}-\frac{\left(2 l_{N-1}+N-3\right)\left(2 l_{N-1}+N-1\right)}{2}-(n+1)^{2}-\left(n+\frac{1}{2}\right)\left(-1+\sqrt{1+\left(2 l_{N-1}+N-3\right)\left(2 l_{N-1}+N-1\right)}\right)}{2 n+2 l_{N-1}+N-1}\right]^{2} .
\end{aligned}
$$

In spherical coordinates, the non-relativistic energy spectrum reported for Yukawa potential is in agreement with the [29].

\subsection{Coulomb Potential}

By setting $D_{e}=0$ and $V_{1}=0$ in Equation (1), we can express Coulomb potential as follows

$V_{N}(r)=-\frac{V_{0}}{r}$

and the rovibrational energy spectrum for Coulomb potential can be written in the hyperspherical coordinates

$$
\begin{aligned}
& E_{n l_{N-1}}=\frac{\hbar^{2} \delta^{2}}{2 \mu}\left[\frac{\left(2 l_{N-1}+N-3\right)\left(2 l_{N-1}+N-1\right)}{4}-\frac{2 \mu V_{0}}{\hbar^{2} \delta}\right] \\
& -\frac{\hbar^{2} \delta^{2}}{2 \mu}\left[\frac{\frac{2 \mu V_{0}}{\hbar^{2} \delta}-\frac{\left(2 l_{N-1}+N-3\right)\left(2 l_{N-1}+N-1\right)}{2}-(n+1)^{2}-\left(n+\frac{1}{2}\right)\left(-1+\sqrt{1+\left(2 l_{N-1}+N-3\right)\left(2 l_{N-1}+N-1\right)}\right)}{2 n+2 l_{N-1}+N-1}\right]^{2} .
\end{aligned}
$$

In three dimensions, this energy equation matches with the ones studied in [29]. On the other hand, if the screening parameter approaches zero $(\delta \rightarrow 0)$ and also $D_{e}=0, V_{1}=0$, Equation (32) transforms to the energy spectrum obtained by Dong et al.[24] irrespective of $l_{N-1}$.

Table 1. Comparison of energy eigenvalues $\left(E_{n, l}\right)$ for Hellmann potential in $N=3$ dimensional system

\begin{tabular}{|c|cccc|}
\hline \multirow{2}{*}{ States } & $V_{0}=2$ & $V_{1}=-1$ & & \\
& $\delta$ & AIM & NU[14] & AP[14] \\
\hline $1 \mathrm{~s}$ & 0.001 & -2.250500 & -2.24900 & -2.248981 \\
& 0.005 & -2.252506 & -2.24501 & -2.244993 \\
& 0.010 & -2.255025 & -2.24005 & -2.240030 \\
$2 \mathrm{~s}$ & 0.001 & -0.563001 & -0.561502 & -0.561502 \\
& 0.005 & -0.565025 & -0.557550 & -0.557549 \\
& 0.010 & -0.567600 & -0.552697 & -0.552697 \\
$2 \mathrm{p}$ & 0.001 & -0.562250 & -0.561502 & -0.561502 \\
& 0.005 & -0.561256 & -0.557541 & -0.557541 \\
& 0.010 & -0.560025 & -0.552664 & -0.552664 \\
$3 \mathrm{~s}$ & 0.001 & -0.250502 & -0.249004 & -0.249004 \\
& & & &
\end{tabular}




\begin{tabular}{|l|llll|}
\multirow{3}{*}{$3 p$} & 0.005 & -0.252556 & -0.245111 & -0.245110 \\
& 0.010 & -0.255225 & -0.240435 & -0.240435 \\
& 0.001 & -0.250168 & -0.249004 & -0.249004 \\
& 0.005 & -0.250867 & -0.245103 & -0.245102 \\
& 0.010 & -0.251802 & -0.240404 & -0.240404 \\
\hline
\end{tabular}

So as to exhibit the accuracy of the asymptotic iteration method, we compute the non-relativistic energy eigenvalues in three dimensional space for various quantum numbers with the different values of $\delta$. In these calculations, $\hbar=2 \mu=1$ are taken. The calculated results are presented in Tables 1-3. It is seen that our results provide a good agreement with those obtained by using the Nikifirov-Uvarov(NU) and amplitudephase(AP) method.

Table 2. Comparison of energy eigenvalues $\left(E_{n, l}\right)$ for Yukawa potential in $N=3$ dimensional system

\begin{tabular}{|c|cccc|}
\hline \multirow{2}{*}{ States } & $V_{0}=0$ & $V_{1}=-3$ & & \\
& $\delta$ & AIM & NU[14] & AP[14] \\
\hline $1 \mathrm{~s}$ & 0.001 & -2.248500 & -2.248500 & -2.247001 \\
& 0.005 & -2.242506 & -2.242506 & -2.235037 \\
& 0.010 & -2.235025 & -2.235025 & -2.220149 \\
$2 \mathrm{~s}$ & 0.001 & -0.561001 & -0.561001 & -0.559506 \\
& 0.005 & -0.555025 & -0.555025 & -0.547649 \\
& 0.010 & -0.547600 & -0.547600 & -0.533091 \\
$2 \mathrm{p}$ & 0.001 & -0.560250 & -0.560250 & -0.559505 \\
& 0.005 & -0.551256 & -0.551256 & -0.547624 \\
& 0.010 & -0.540025 & -0.540025 & -0.532993 \\
$3 \mathrm{~s}$ & 0.001 & -0.248502 & -0.248502 & -0.247013 \\
& 0.005 & -0.242556 & -0.242556 & -0.235332 \\
& 0.010 & -0.235225 & -0.235225 & -0.221306 \\
$3 \mathrm{p}$ & 0.001 & -0.248168 & -0.248168 & -0.247012 \\
& 0.005 & -0.240867 & -0.240867 & -0.235308 \\
& 0.010 & -0.231802 & -0.231803 & -0.221212 \\
\hline
\end{tabular}

Table 3. Comparison of energy eigenvalues $\left(E_{n, l}\right)$ for Coulomb potential in $N=3$ dimensional system

\begin{tabular}{|c|cccc|}
\hline \multirow{2}{*}{ States } & $V_{0}=3$ & $V_{1}=0$ & & \\
& $\delta$ & AIM & NU[14] & AP[14] \\
\hline \multirow{7}{*}{$1 \mathrm{~s}$} & 0.001 & -2.251500 & -2.251500 & -2.250000 \\
& 0.005 & -2.257506 & -2.257506 & -2.250000 \\
& 0.010 & -2.265025 & -2.265025 & -2.250000 \\
$2 \mathrm{~s}$ & 0.001 & -0.564001 & -0.564001 & -0.562500 \\
& 0.005 & -0.570025 & -0.570025 & -0.562500 \\
& 0.010 & -0.577600 & -0.577600 & -0.562500 \\
$2 \mathrm{p}$ & 0.001 & -0.563250 & -0.563250 & -0.562500 \\
& 0.005 & -0.566256 & -0.566256 & -0.562500
\end{tabular}




\begin{tabular}{|l|llll|}
\multirow{3}{*}{$3 \mathrm{~s}$} & 0.010 & -0.570025 & -0.570025 & -0.562500 \\
& 0.001 & -0.251502 & -0.251502 & -0.250000 \\
& 0.005 & -0.257556 & -0.257556 & -0.250000 \\
$3 \mathrm{p}$ & 0.010 & -0.265225 & -0.265225 & -0.250000 \\
& 0.001 & -0.251168 & -0.251168 & -0.250000 \\
& 0.005 & -0.255867 & -0.255867 & -0.250000 \\
& 0.010 & -0.261802 & -0.261803 & -0.250000 \\
\hline
\end{tabular}

\section{CONCLUSION}

In this work, the problem of solving the N-dimensional Schrödinger equation with Hellmann plus Kratzer potential for any $l_{N-1}$-state have been studied from the point of view the asymptotic iteration formalism. To achieve this, we have applied a proper approximation scheme which is called the Greene-Aldrich approximation. Thus, the approximate analytic expressions for the energy eigenvalues and the corresponding wavefunctions of quantum system with Hellmann plus Kratzer potential have been built in hyperspherical coordinates. In addition, for the special cases of the potential parameters, we have also found the energy spectra for Hellmann, the modified Kratzer, Yukawa, Coulomb potentials in hyperspherical coordinates. We have computed the numerical energy eigenvalues for Hellmann, Yukawa, Coulomb potentials in $N=3$ dimensional systems. The results obtained within the framework of asymptotic iteration approach consistent with the other methods in literature.

We highlight that asymptotic iteration formalism is a robust computational method to obtain the approximate solutions of the $\mathrm{N}$-dimensional non-relativistic wave equation for a particle in central potential field. It should be emphasized that these $\mathrm{N}$-dimensional results allow us to the comprehension of a general treatment of this problem. Moreover, we can also obtain directly the energy equations in two and three dimensions from the $\mathrm{N}$-dimensional results for the system.

\section{CONFLICTS OF INTEREST}

No conflict of interest was declared by the author.

\section{REFERENCES}

[1] Greene, R.L., Aldrich, C., "Variational wave functions for a screened Coulomb potential", Physical Review A, 14 (6): 2363-2366, (1976).

[2] Ciftci, H., Hall, R.L., Saad, N., "Asymptotic iteration method for eigenvalue problems”, Journal of Physics A: Mathematical and General, 36(47): 11807-11816, (2003).

[3] Ciftci, H., Hall, R.L., Saad, N., "Construction of exact solutions to eigenvalue problems by the asymptotic iteration method", Journal of Physics A: Mathematical and General, 38 (5): 1147-1155, (2005).

[4] Ciftci, H., Hall, R.L., Saad, N., "Iterative solutions to the Dirac equation", Physical Review A, 72 (2): 022101-7, (2005).

[5] Louck, J.D., Shaffer, W.H., "Generalized orbital angular momentum and the n-fold degenerate quantum mechanical oscillator: Part I the twofold degenerate oscilator", Journal of Molecular Spectroscopy, 4 (1-6): 285-297, (1960). 
[6] Louck, J.D,"Generalized orbital angular momentum and the n-fold degenerate quantum mechanical oscillator : Part II the n-fold degenerate oscillator" Journal of Molecular Spectroscopy, 4 (1-6): 298-333, (1960).

[7] Louck, J.D,"Generalized orbital angular momentum and the n-fold degenerate quantum mechanical oscillator : Part III radial integrals” Journal of Molecular Spectroscopy, 4 (1-6): 334$341,(1960)$.

[8] Chatterjee, A., "Large-N expansions in quantum mechanics, atomic physics and some $\mathrm{O}(\mathrm{N})$ invariant systems", Physics Reports, 186 (6): 249-370, (1990).

[9] Hellmann, H., “ A new approximation method in the problem of many electrons”, The Journal of Chemical Physics, 3 (1): 61, (1935).

[10] Hellmann, H., Kassatotchkin, W., "Metallic Binding According to the combined approximation procedure", The Journal of Chemical Physics, 4(5): 324-325, (1935).

[11] Kratzer, A.,“ Die ultraroten rotationsspektren der halogenwasserstoffe”, Zeitschrift für Physik, 3 (5): 289-307, (1920).

[12] Hassanabadi H., Rahimov H., Zarrinkamar S., "Approximate solutions of Klein-Gordon equation with Kratzer potential”, Advances in High Energy Physics, (2011).

[13] Ikot A.N., Etuk S.E., Hassanabadi H., Maghsoodi E., Zarrinkamar S., "Dirac equation under Hellmann potential as pseudoscalar potential”, Indian Journal of Physics, 89: 289-294, (2015).

[14] Hamzavi, M., Thylwe, K.E, Rajabi, A.A.," Approximate bound states solutions of the Hellmann potential", Communications in Theoretical Physics, 60(1): 1-8, (2013).

[15] Oyewumi, K.J., "Realization of the spectrum generating algebra for the generalized Kratzer potentials", International Journal of Theoretical Physics, 49(6): 1302-1316, (2010).

[16] Dutt, R., Mukherji, U., Varshni, Y.P.," An improved calculation for screened Coulomb potentials in Rayleigh-Schrodinger perturbation theory", Journal of Physics A: Mathematical and General, 18: 1379-1388, (1985).

[17] Vrscay, E.R.,“ Hydrogen atom with a Yukawa potential: Perturbation theory and continuedfractions-Padé approximants at large order”, Physical Review A, 33(2): 1433-1436, (1986).

[18] Stubbins, C., “ Bound states of the Hulthén and Yukawa potentials”, Physical Review A, 48(1): 220-227, (1993).

[19] Adamowski, J.,“ Bound eigenstates for the superposition of the Coulomb and the Yukawa potentials", Physical Review A, 31(1): 43-50, (1985).

[20] Simons, G., Parr, R.G., Finlan, J.M., “ New alternative to the Dunham potential for diatomic molecules”, The Journal of Chemical Physics, 59(6): 3229-3234, (1973).

[21] Molski, M, Konarski, J.,"Extended Simons-Parr-Finlan approach to the analytical calculation of the rotational-vibrational energy of diatomic molecules", Physical Review A, 47(1): 711-714, (1993).

[22] Pliva, J., “A closed rovibrational energy formula based on a modified Kratzer potential”, Journal of Molecular Spectroscopy, 193(1): 7-14, (1999). 
[23] Edet, C.O., Okorie, K.O., Louis, H., Nzeata-Ibe, N., “ Any l-state solutions of the Schrödinger equation interacting with Hellmann-Kratzer potential model", Indian Journal of Physics, (2019).

[24] Dong, S.H., Sun, G.H., "The Schrödinger equation with a Coulomb plus inverse-square potential in D-dimensions", Physica Scripta, 70(2-3): 94-97, (2004).

[25] Durmus, A., "Nonrelativistic treatment of diatomic molecules interacting with a generalized Kratzer potential in hyperspherical coordinates", Journal of Physics A: Mathematical and General, 44(15): 155205-14, (2011).

[26] Ikdhair, S.M., Sever, R., "Exact solutions of the pseudo-Coulomb potential plus ring-shaped potential in the D-dimensional Schrödinger equation by the Nikiforov-Uvarov method", arXiv: quany-ph/0703042v1 2007; 1-15.

[27] Durmus, A.," Approximate treatment of the Dirac equation with hyperbolic potential function", Few-Body Systems, 59(7), (2018).

[28] Gradshteyn, I.S., Ryzhik, I.M., "Tables of Integrals, Series and Products”. Academic Press, New York, (2000).

[29] Ebomwonyi, O., Onate, C.A., Onyeaju, M.C., Ikot, A.N., "Any l-state solutions of the Schrödinger equation interacting with Hellmann-generalized Morse potential model", Karbala International Journal of Modern Science, 3(1): 59-68, (2017).

\section{APPENDIX: FORMULATION OF THE ASYMPTOTIC ITERATION APPROACH}

Asymptotic iteration method is capable of solving second-order homogeneous linear differential equations of the form

$y^{\prime \prime}(r)=\lambda_{0}(r) y^{\prime}(r)+s_{0}(r) y(r)$

in which $\lambda_{0}(r)$ and $s_{0}(r)$ functions in $C_{\infty}(a, b)$ are sufficiently differentiable. The general solution of Equation (A.1) can be obtained in the following form

$y(r)=\exp \left(-\int^{r} \alpha\left(r^{\prime}\right) d r^{\prime}\right)\left[C_{2}+C_{1} \int^{r} \exp \left(\int^{r^{\prime}}\left[\lambda_{0}(\tau)+2 \alpha(\tau)\right] d \tau\right) d r^{\prime}\right]$

For sufficiently large $k$,

$\frac{s_{k}(r)}{\lambda_{k}(r)}=\frac{s_{k-1}(r)}{\lambda_{k-1}(r)}=\alpha(r)$

in which

$\lambda_{k}(r)=\lambda_{k-1}^{\prime}(r)+s_{k-1}(r)+\lambda_{0}(r) \lambda_{k-1}(r)$

$s_{k}(r)=s_{k-1}^{\prime}(r)+s_{0}(r) \lambda_{k-1}(r)$.

If the eigenvalue problem has exact analytical solutions, the termination condition Equation (A.3), or equivalently 
$\delta_{k}(r)=\lambda_{k}(r) s_{k-1}(r)-\lambda_{k-1}(r) s_{k}(r)=0$

produces, at each iteration, an expression that is independent of $r$. It is note that $k$ displays the iteration number. Physically meaningful solution of Equation (A.1) is provided by the first term of Equation (A.2) not the second term, so we can use the first term as the wavefunction generator

$y(r)=C_{2} \exp \left(-\int^{r} \frac{s_{k}\left(r^{\prime}\right)}{\lambda_{k}\left(r^{\prime}\right)} d r^{\prime}\right)$

in which $C_{2}$ denotes the integrant constant which can be determined by normalization. It should be mentioned that the details concerning this method can be found in [2-4]. 